\title{
Biocompatibility and water durability of alumina-zirconia ceramics blended with microsized HA particles
}

\author{
Yumi TANAKA,,${ }^{* * *, \dagger}$ Masato YOSHIDA, ${ }^{*, * * *}$ Miho NAKAMURA, ${ }^{*}$ Akiko NAGAI, ${ }^{*}$ \\ Kazuaki HASHIMOTO, ${ }^{* * *}$ Yoshitomo TODA*** and Kimihiro YAMASHITA* \\ *Department of Inorganic Materials, Institute of Biomaterials and Bioengineering, Tokyo Medical and Dental University, \\ 2-3-10 Kanda-surugadai, Chiyoda, Tokyo 101-0062 \\ **Department of applied chemistry, Faculty of Engineering, Kyushu University, 744 Motooka, Nishi-ku, Fukuoka 819-0395 \\ ${ }^{* * *}$ Department of Life and Environmental Sciences, Faculty of Engineering, Chiba Institute of Technology, \\ 2-17-1 Tsudanuma, Narashino, Chiba 275-0016
}

To improve the bioactivity of alumina containing yttria stabilized zirconia (Al-YSZ), composite ceramics consisting of Al-YSZ and 0-10 wt \% of hydroxyapatite (HA) were prepared. The XRD patterns of sintered disks with an HA content above 3 wt \% had monoclinic and cubic YSZ phases and a $\beta$-tricalcium phosphate phase. Although the Vickers hardness decreased as the HA content increased, all the prepared ceramics had the same or greater hardness $(1000-1300 \mathrm{Hv}$ with a load of $0.49 \mathrm{~N})$ compared to typical YSZ ceramics $(900-1000 \mathrm{Hv})$ commonly used in clinics. Furthermore, the bonelike apatite formation ability in simulated body fluid, which was examined as a screening of bioactivity, suggested that all the surfaces of Al-YSZ ceramics blending with a small amount of HA may possess the ability to connect bone. After the accelerated low temperature degradation (LTD) test performed in normal saline at $120^{\circ} \mathrm{C}$ for five days using composite ceramics with the $\mathrm{HA}$ content of $0,1,3$, and $10 \mathrm{wt} \%$, the tetragonal zirconia phase of all composite ceramics were partially transformed into the monoclinic phase with a transition ratio of ca. 16-18 mol \% (for $\mathrm{HA}=0$ and $10 \mathrm{wt} \%$ ) and ca. 4-5 mol \% (for $\mathrm{HA}=1$ and $3 \mathrm{wt} \%$ ). Blending microsized $\mathrm{HA}$ powder in Al-YSZ ceramics has both positive and negative effects on the LTD durability of Al-YSZ ceramics. An advantage is that calcium ion substitution in YSZ seems to stabilize the tetragonal (or cubic) zirconia phases, but the increased surface area exposed to the solution is a disadvantage.

(C2010 The Ceramic Society of Japan. All rights reserved.

Key-words : Alumina-zirconia ceramics, Biomaterial, Composite, Hydroxyapatite, Low temperature degradation (LTD)

[Received February 16, 2010; Accepted April 15, 2010]

\section{Introduction}

Tetragonal zirconia, which is the high temperature phase between $1117-2300^{\circ} \mathrm{C}$, shows a high hardness, toughness, and excellent ultimate strength due to its unique property of a pressure induced martensitic phase transition. ${ }^{1)}$ Because the tetragonal phase is stabilized under lower temperature when several molar percent of certain cations (e.g. $\mathrm{Ca}^{2+}, \mathrm{Mg}^{2+}$ and most rare-earth ions) are homogeneously doped into $\mathrm{Zr}^{4+}$, a series of stabilized zirconia is widely used in load bearing applications. In particular, yttria stabilized tetragonal zirconia (YSZ) is one of the most important biomaterials, and is utilized as artificial teeth and femoral head prosthesis due to the good biocompatibility and stability at body temperature. However, biomedical applications of YSZ are very limited because it cannot directly bond with living hard tissues. $^{2)}$

Numerous research has focused on developing YSZ ceramics with bone connecting ability (bioactivity) because bioceramics possessing excellent mechanical properties and sufficient bioactivity should lead to various applications e.g. ceramic bolts to join bones, a metal-free artificial joint system for knees, shoulders, fingers, and ankles. According to Uchida et al., surface modifications with hydroxyl ions, which act as nucleation

\footnotetext{
Corresponding author: Y. Tanaka; E-mail: yutanaka@ cstf.kyushuu.ac.jp
}

sites for new bones, is one of the most attractive techniques to provide bioactivity to various biomaterials. ${ }^{3)}$ However, in the case of YSZ, this technique is at a competitive disadvantage in terms of speedier "low temperature degradation" (LTD); ;) $^{4}$ some of the tetragonal phase turns into the monoclinic phase during chemical treatment using strong acid or alkali solutions, which seriously reduces the mechanical strength. Another effective technique reported to provide bioactive YSZ ceramics is blending with powdered bioactive ceramics. ${ }^{5)}$ Whereas insufficient densification of composite ceramics frequently causes a reduction in mechanical strength and a promoted LTD in an in vivo environment, the blending technique appears to have practical advantages compared to chemical modification because of its simple procedure. Hence, our research strives to provide a bioactive YSZ composite with sufficient mechanical strength and LTD durability by HA powder blending. To date, we have demonstrated that blending with a small amount of spherical, microsized HA powders in YSZ below $10 \mathrm{wt} \%$ effectively provides bonelike apatite forming ability to YSZ in simulated body fluid (SBF) while simultaneously inhibiting a great decrease of the hardness. ${ }^{6}$ Herein alumina containing yttria stabilized zirconia (Al-YSZ) has a higher flexural strength than YSZ due to the small sized grain growth during sintering, $\left.{ }^{7}\right)$ Al-YSZ was used as the matrix. To reduce the adverse effect of HA particles on the densification of Al-YSZ during sintering, only a small amount of HA (below $10 \mathrm{wt} \%$ ) was blended also in this study. 


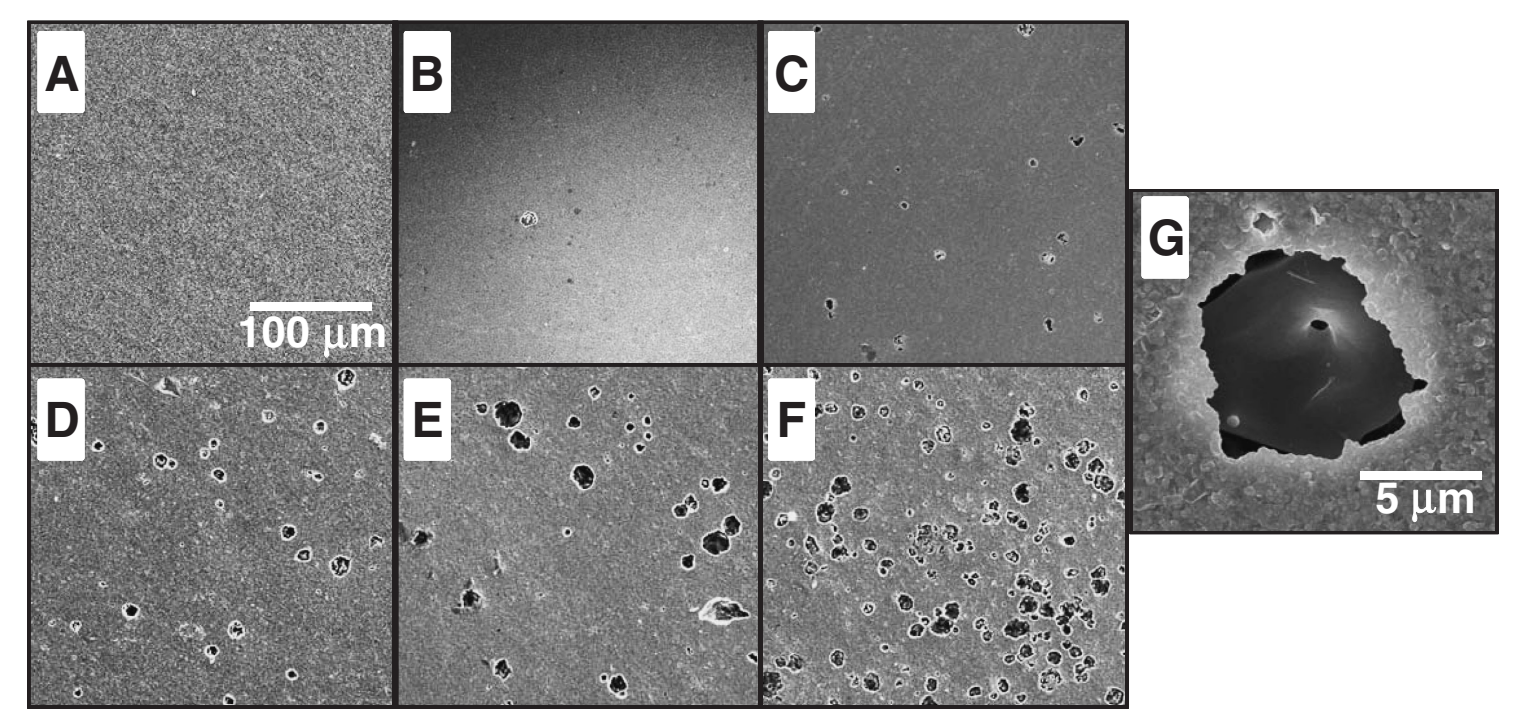

Fig. 1. SEM photographs on the surface of A) AZ-H(0), B) AZ-H(0.1), C) AZ-H(0.5), D) AZ-H(1), E) AZ-H(3), and F) $\mathrm{AZ}-\mathrm{H}(10)$ sintered at $1450^{\circ} \mathrm{C}$ for $1 \mathrm{~h}$ in air. Photograph $\mathrm{G}$ shows a magnified image around a pore.

\section{Materials and methods}

After the homogeneous blending of $0,0.1,0.5,1,3$, and $10 \mathrm{wt} \%$ of HA powder (consisting of spherical particles measur-

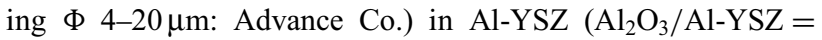
$20 \mathrm{wt} \%$ and $\mathrm{Y}_{2} \mathrm{O}_{3} / \mathrm{YSZ}=3 \mathrm{~mol} \%$ : TZ-3Y20AB, Tosoh Co.), composite powders were compacted into green disks by uniaxial pressing under $150 \mathrm{MPa}$ and sintering into ceramics at $1450^{\circ} \mathrm{C}$ for $1 \mathrm{~h}$ in air (herein the obtained ceramics are identified as AZ$\mathrm{H}(x)(x=0-10))$. For comparison, ceramic disks of single phase $\mathrm{HA}$ and $\mathrm{YSZ}\left(\mathrm{Y}_{2} \mathrm{O}_{3} / \mathrm{YSZ}=3 \mathrm{~mol} \%\right.$; TZ-3YB, Tosho Co. $)$ were prepared at $1250^{\circ} \mathrm{C}$ for $2 \mathrm{~h}$ under streaming steam (for HA) and $1400^{\circ} \mathrm{C}$ for $1 \mathrm{~h}$ under air (for YSZ). Morphologies of the obtained ceramic surfaces were observed by scanning electron microscopy (SEM) using a HITACHI S-3400NX microscope, and the crystal phases of the ceramic disks were characterized by $2 \theta / \theta$ X-ray diffractometry (XRD) using a Philips PW-1700 diffractometer. Mechanical strengths of AZ-H $(x)$ ceramics were compared to those of HA and YSZ as a function of Vickers hardness. The measurements for each disk were repeated 12 times a disk under a load of $0.49 \mathrm{~N}$ using a Shimazu DUH200.

Instead of directly investigating the bioactivity of ceramics using an expensive and long-term animal test, the bioactivity was roughly screened as a function of bonelike apatite formation ability in simulated body fluid (an inorganic solution with the same ionic concentrations as human plasma: SBF). ${ }^{8)}$ Each ceramic disk was aged in $10 \mathrm{ml}$ of $1.5 \mathrm{SBF}$ (1.5 times as dense as normal SBF, which is an accelerated condition for bonelike apatite formation) at $37^{\circ} \mathrm{C}$ for $1-5$ days. After gently washing and drying naturally, the deposits on the aged ceramics were observed by SEM.

The influence of HA blending on LTD durability of Al-YSZ ceramics was estimated as a function of the tetragonal/ monoclinic transition ratio in normal saline using disks of $\mathrm{AZ}-\mathrm{H}(0),(1),(3)$, and (10); aging temperature and time were $120^{\circ} \mathrm{C}$ and $1-5$ days. The transition ratio was calculated by the XRD patterns using Garvie and Nicholson's equation: ${ }^{9)}$

$$
T_{\mathrm{t}-\mathrm{m}}=\left(I_{\mathrm{m}(111)}+I_{\mathrm{m}(11 \overline{1})}\right) /\left(I_{\mathrm{m}(111)}+I_{\mathrm{m}(11 \overline{1})}+I_{\mathrm{t}(111)}\right)
$$

where $T_{\mathrm{t}-\mathrm{m}}$ is the molar translation ratio from the tetragonal to the monoclinic phase. $I_{\mathrm{m}(h k l)}$ and $I_{\mathrm{t}(h k l)}$ are the XRD peak

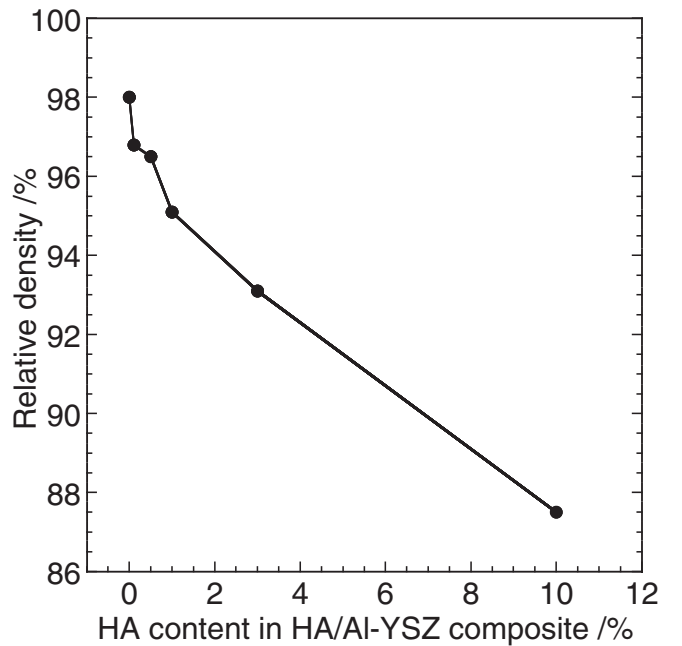

Fig. 2. Influence of $\mathrm{HA}$ content on the relative density of $\mathrm{AZ}-\mathrm{H}(x)$ ceramics sintered at $1450^{\circ} \mathrm{C}$ for $1 \mathrm{~h}$ in air.

intensities by $(h k l)$ plane of the monoclinic and tetragonal phases, respectively.

\section{Results and discussion}

\subsection{Characterization of the composite ceramics}

Sintering Al-YSZ compacts blended with HA particles caused spherical pores to form (Fig. 1A-F) where shrunken particles appeared to be embedded (Fig. 1G). The pores increased with the HA content, resulting in a monotonic decrease in the relative density from $98 \%$ of $\mathrm{AZ}-\mathrm{H}(0)$ to $87.5 \%$ of $\mathrm{AZ}-\mathrm{H}(10)$ (Fig. 2). Moreover, HA blending influenced crystal phases in the obtained ceramics as shown in Fig. 3; monoclinic and cubic zirconia phases were found in addition to the starting phases of Al-YSZ (tetragonal zirconia and $\alpha$-alumina), and $\beta$-tricalcium phosphate ( $\beta$-TCP) phase was detected instead of HA in the sinters with HA content above $3 \mathrm{wt} \%$. $\beta$-TCP was probably generated via the decomposition of $\mathrm{HA}\left(\mathrm{Ca}_{10}\left(\mathrm{PO}_{4}\right)_{6}(\mathrm{OH})_{2} \rightarrow 3 \mathrm{Ca}_{3}\left(\mathrm{PO}_{4}\right)_{2}+\right.$ $\mathrm{CaO}+\mathrm{H}_{2} \mathrm{O}$ ), while $\mathrm{CaO}$ temporarily derived from decomposition likely gave rise to the local transformation of the tetragonal zirconia phase into the cubic phase; the lack of XRD peaks of 


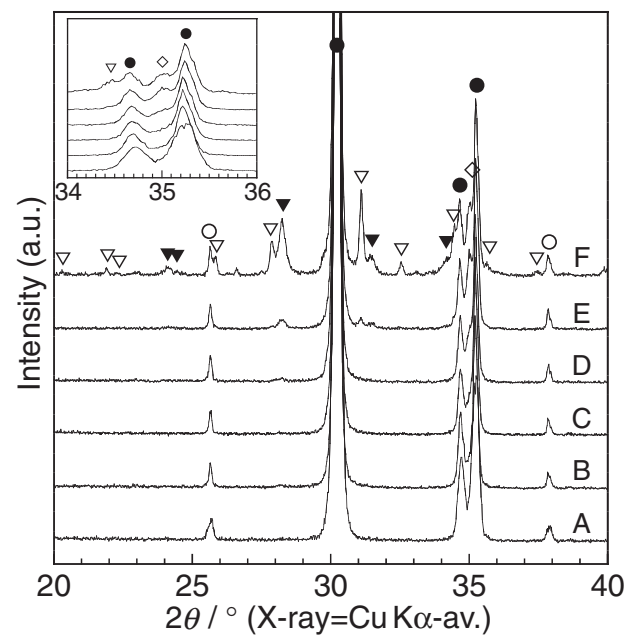

Fig. 3. XRD patterns of A) AZ-H(0), B) AZ-H(0.1), C) AZ-H(0.5), D) AZ-H(1), E) AZ-H(3), and F) AZ-H(10) sintered at $1450^{\circ} \mathrm{C}$ for $1 \mathrm{~h}$ in air. Inset is a magnified image of the diffractions between $2 \theta=34-36^{\circ}$ where several peaks seem to overlap. Diffraction peaks originating in monoclinic, tetragonal, and cubic zirconia are identified by solid triangle, solid circle, and white diamond, respectively. a-Alumina and b-TCP are identified by a white circle and triangle, respectively.

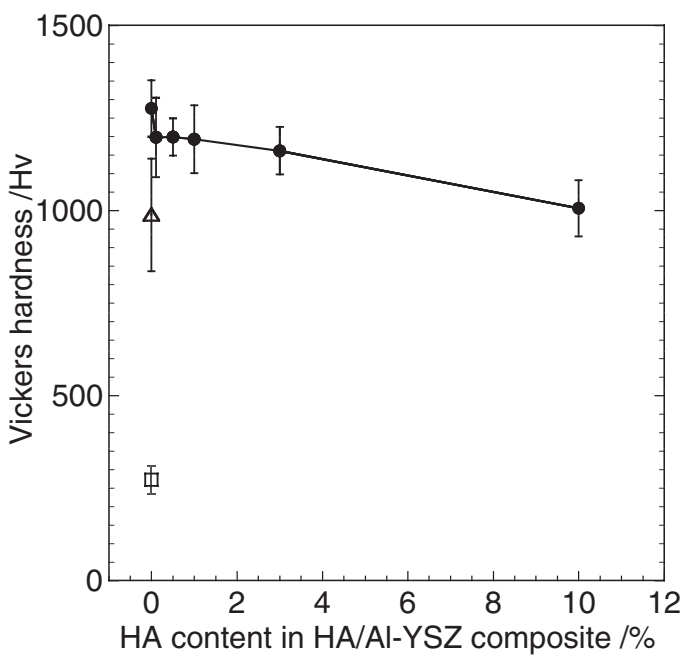

Fig. 4. Influence of HA content on the Vickers hardness of AZ-H(x) ceramics sintered at $1450^{\circ} \mathrm{C}$ for $1 \mathrm{~h}$ in air. Hardnesses of $\mathrm{HA}$ and YSZ ceramics (denoted by a white square and triangle, respectively) are plotted for comparison.

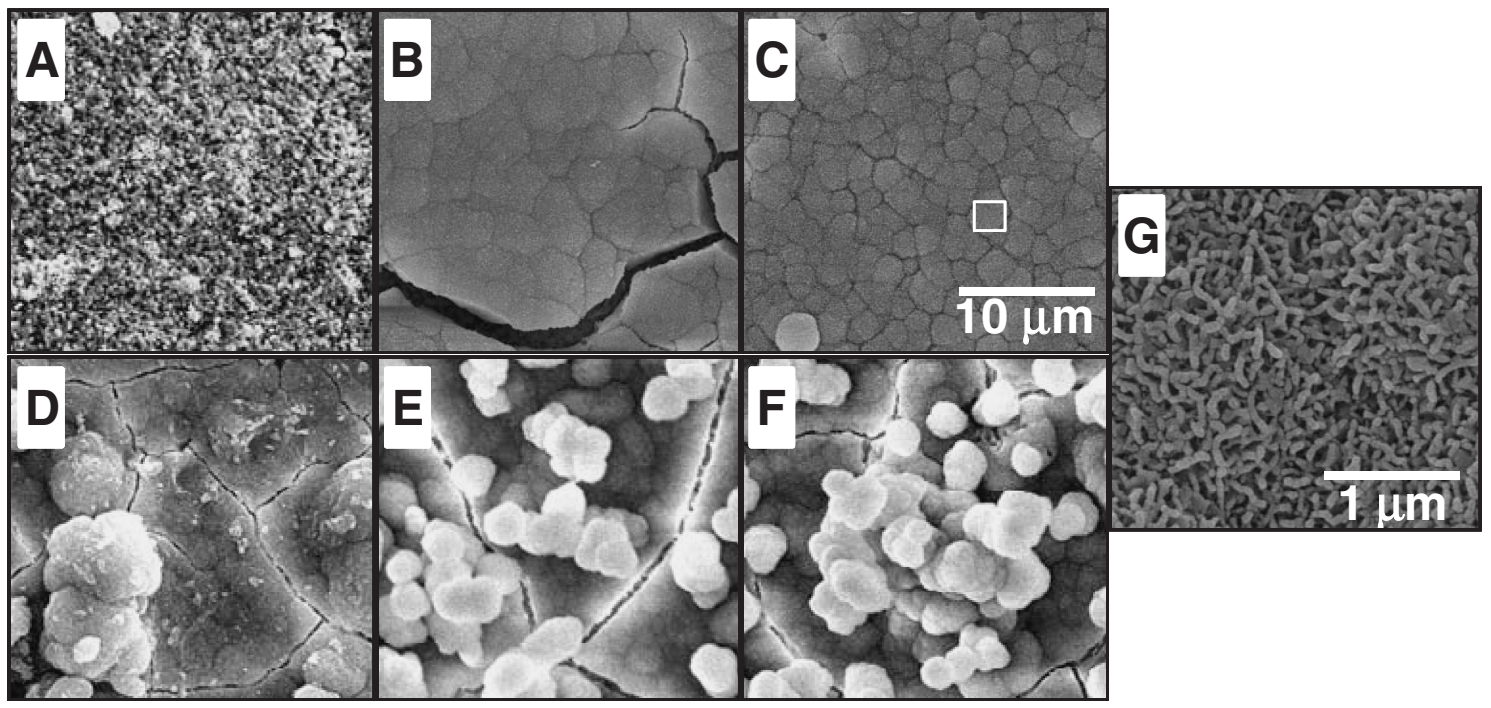

Fig. 5. SEM photographs on the surface of A) AZ-H(0), B) AZ-H(0.1), C) AZ-H(0.5), D) AZ-H(1), E) AZ-H(3), and F) AZ-H(10) sintered at $1450^{\circ} \mathrm{C}$ for $1 \mathrm{~h}$ in air after immersing for five days in $1.5 \mathrm{SBF}$ at $37^{\circ} \mathrm{C}$. Photograph $\mathrm{G}$ shows a magnified image of the area marked in photograph $\mathrm{C}$.

$\mathrm{CaO}$ in Fig. 3 indicated that almost all $\mathrm{CaO}$ was used to stabilize the cubic phase by forming calcia-zirconia solid solution. Although the origin of the formation of the monoclinic phase remains unclear, one plausible origin is that stress generation around the boundaries between HA and Al-YSZ with different thermal expansion behaviors induces a martensitic phase transition from the tetragonal phase to the monoclinic in the heating or cooling process during sintering. Whereas obvious peaks of impurities were not detected in XRD patterns due to the very small amount of blending, the same reactions were also expected for AZ-H(0.1)-(1).

As shown in Fig. 4, Vickers hardness of AZ-H(x) ceramics initially decreased by ca. $6 \%$ (from 1275 to $1195 \mathrm{Hv}$ ) between $x=0$ and 0.1 , followed by a continuous, relatively gradual decrease as the HA content increased up to $x=10(1010 \mathrm{Hv}$ corresponds to the decrement of ca. 20\%). An adverse effect of HA blending caused the decreased relative density of the sinters with micropores. However, all of the values were much higher than that of HA ceramics, and were equal or higher compared to the typical value of YSZ ceramics used in clinics.

\subsection{Bonelike apatite formation ability}

After immersing for five days in $1.5 \mathrm{SBF}$, the surfaces of AZ$\mathrm{H}(x)$ ceramics were completely covered with worm-like deposits, except the surface of $x=0$ (Fig. 5); the crystal phase of the deposits was identified as HA with a low crystallinity by comparing the XRD patterns of AZ-H $(x)$ ceramics before and after immersion (Fig. 6). This finding suggests that even a very small amount of $0.1 \mathrm{wt} \%$ blending of HA has the potential to provide bone connecting ability to bioinert Al-YSZ ceramics. As 


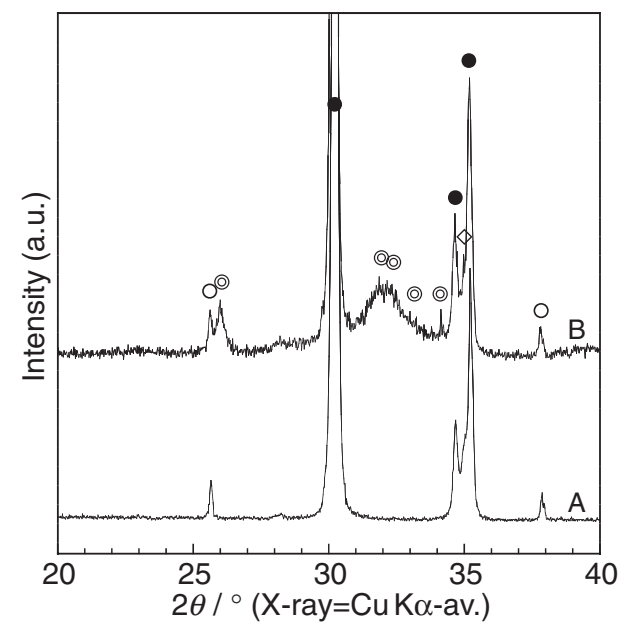

Fig. 6. XRD patterns of ceramics of $\mathrm{AZ}-\mathrm{H}(1)$ sintered at $1450^{\circ} \mathrm{C}$ for $1 \mathrm{~h}$ in air, A) before and B) after immersing for five days in 1.5SBF at $37^{\circ} \mathrm{C}$. Diffraction peaks originating in tetragonal and cubic zirconia are identified by a solid circle and white diamond, respectively. a-Alumina and HA are identified by white and double circles, respectively.

described in the previous section, HA particles blended in AlYSZ compacts likely decomposed with forming shrunken particles of $\beta$-TCP embedded in micropores. Judging from the relatively high water solubility of $\beta$-TCP, ${ }^{10)}$ the particles in AZ$\mathrm{H}(x)$ ceramics should dissolve in SBF accompanied by a local increase in the calcium ion concentration within the pores, which probably acts as a trigger for nucleation. The pores filled with deposits should play the role of wedges for following crystal growth of bonelike apatite. On the other hand, $\mathrm{CaO}$ probably generated during decomposition of HA should not have much influence on bonelike apatite formation because the complete incorporation of $\mathrm{CaO}$ in zirconia phase is expected in the present $\mathrm{AZ}-\mathrm{H}(x)$ ceramics as described in the previous section.

\subsection{Low temperature degradation durability}

During the accelerated LTD test, which was representatively conducted using $\mathrm{AZ}-\mathrm{H}(0),(1)$, (3), and (10), the tetragonal zirconia phase of all composite ceramics was partially transformed into the monoclinic phase with a transition ratio of ca. $16 \mathrm{~mol} \%$ for AZ-H(0), $5 \mathrm{~mol} \%$ for AZ-H(1), $4 \mathrm{~mol} \%$ for AZ$\mathrm{H}(3)$, or $18 \mathrm{~mol} \%$ for AZ-H(10) after aging for five days (Fig. 7). The degradation rates of AZ-H(1) and (3) were slower than that of $\mathrm{AZ}-\mathrm{H}(0)$ as well as that of $\mathrm{AZ}-\mathrm{H}(10)$, demonstrating that the blending of HA both positively and negatively affects LTD durability of Al-YSZ ceramics. The improved LTD durability of Al-YSZ ceramics by blending a small amount of HA is probably because substituting zirconium ion sites with calcium ions supplied from HA during sintering stabilized the tetragonal phase. In contrast, the decreased relative density and partial phase transition to monoclinic zirconia in the starting ceramics caused by the excess HA adversely influenced not only the initial mechanical strength but also the LTD durability. Moreover, these findings suggest the possibility of partially utilizing Ca-stabilized

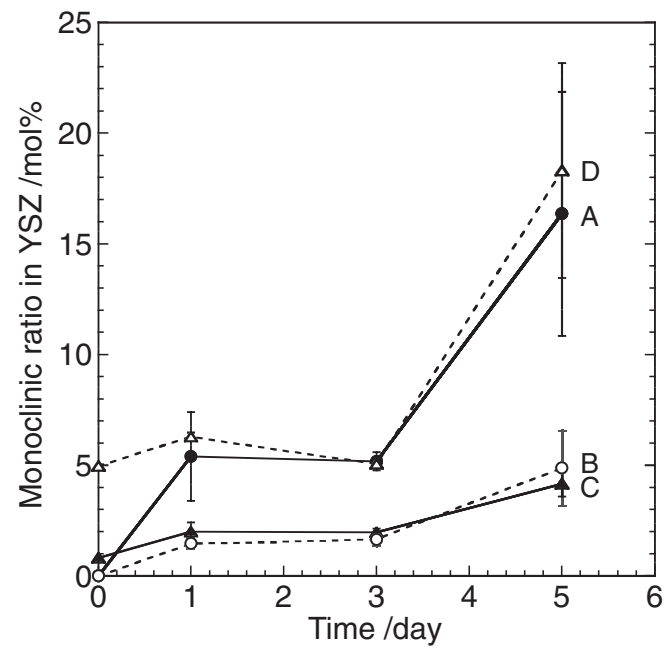

Fig. 7. Change in the tetragonal/monoclinic transition ratio of zirconia phase in A) AZ-H(0), B) AZ-H(1), C) AZ-H(3), and D) AZ-H(10) sintered at $1450^{\circ} \mathrm{C}$ for $1 \mathrm{~h}$ in air during an accelerated LTD test performed in normal saline at $120^{\circ} \mathrm{C}$.

tetragonal or cubic zirconia as well as YSZ as a matrix for zirconia based composite ceramics as a load-bearing bone substitute.

\section{Conclusions}

The findings herein suggest load bearing bioceramics with sufficient mechanical strength, bonelike apatite formation ability in a SBF solution, and good durability for LTD may be realized via a simple procedure of HA blending in Al-YSZ ceramics when the blending content of HA is below $3 \mathrm{wt} \%$.

\section{References}

1) R. C. Garvie, R. H. Hannink and R. T. Pascoe, Nature, 258, 703-704 (1975).

2) C. Pconi and G. Maccauro, Biomaterials, 20, 1-25 (1999).

3) M. Uchida, H. M. Kim, T. Kokubo, M. Nawa, T. Asano and K. Tanaka, J. Biomed. Mater. Res., 60, 227-282 (2002).

4) Y. Tanaka, N. Ukai, K. Nishio and K. Yamashita, Ceram. Eng. Sci. Proc., 29, 239-248 (2008).

5) R. R. Rao and T. S. Kannan, Mater. Sci. Eng., C, 20, 187-193 (2002).

6) M. Inuzuka, S. Nakamura, S. Kishi, K. Yoshida, K. Hashimoto, Y. Toda and K. Yamashita, Solid State Ionics, 172, 509-513 (2004).

7) K. Tsukuma, K. Ueda and M. Shimada, J. Am. Ceram. Soc., 68, c4-c5 (1985).

8) T. Kokubo and H. Kushitani, Biomaterials, 27, 2907-2915 (2006).

9) R. C. Garvie and P. S. Nicholson, J. Am. Ceram. Soc., 55, 303305 (1972).

10) A. Ito, H. Kawamura, S. Miyakawa, P. Layrolle, N. Kanzaki, G. Treboux, K. Onuma and S. Tsutsu, J. Biomed. Mater. Res., Part A, 60, 224-231 (2002). 